\title{
Das Recht auf eine selbstbestimmte Erwerbsbiografie
}

Eine prozedurale Betrachtung von Anpassungs- und Finanzierungsansprüchen für Übergangssituationen ${ }^{1}$

Ein Wandel der Strukturen der Erwerbsarbeit spiegelt sich in der Regel nach einer gewissen Zeit auch in Veränderungen des Arbeits- und Sozialrechts wider. Für solche Anforderungen an das Recht sind unterschiedliche Leitkonzepte vorgeschlagen worden, insbesondere in Hinblick auf eine lebenslauforientierte Gestaltung des Rechts. Der vorliegende Beitrag knüpft an Konzepte für sogenannte Ziehungsrechte und eine "Arbeits- oder Beschäftigungsversicherung " an und entwickelt zunächst den Vorschlag, die hiermit umrissenen Anforderungen an die Regulierung als »Recht auf eine selbstbestimmte Erwerbsbiografie» auf den juristischen Begriff zu bringen (Kapitel 1).

Politische Gestaltung bedarf aber nicht nur solcher Leitbegriffe, sondern auch konkreter Ordnungsvorstellungen. Hierfür muss das subjektive Recht, das der Leitbegriff formuliert, in arbeits- und sozialrechtlichen Ansprüchen konkretisiert werden. Am Beispiel der Errungenschaften und Lücken des geltenden Rechts wird deshalb aufgezeigt, dass in diesem Zusammenhang die Ansprüche auf betriebliche Anpassung und Konfliktlösung systematisch getrennt von den Ansprüchen auf Finanzierung zu behandeln sind (Kapitel 2). Denn die beiden Arten von Ansprüchen unterscheiden sich insbesondere darin, in welchen Verfahren sie durchgesetzt werden können. Die Rede über »Recht « muss aber immer mit der Rede über »Verfahren « verbunden werden. In dem Beitrag wird daher die These vertreten, dass das Recht auf eine selbstbestimmte Erwerbsbiografie einer besonderen prozeduralen Ausgestaltung der damit verbundenen Ansprüche bedarf. Nur die Verbindung entsprechender Ansprüche mit reflexiv wirkenden Verfahren vermag dem Recht tatsächlich Geltung/Durchsetzungskraft zu verschaffen.

1 Der Beitrag ist Ergebnis des Projekts »SozRA« unter Leitung von Eva Kocher und Felix Welti, das von der Hans-Böckler-Stiftung gefördert wurde. Die Ergebnisse erscheinen im Nomos-Verlag (Kocher et al. 2013). In Hinblick auf das »Recht auf Weiterbildung " wurden einige der hier diskutierten Überlegungen auch in Kocher, Welti 2013 konkretisiert. Dank für die Diskussion gebührt Felix Welti, dem Projektteam und dem beratenden ExpertInnenkreis, namentlich Günther Schmid. Den anonymen Gutachterinnen und Gutachtern sei für konstruktives Feedback und Hinweise gedankt! 


\section{Arbeits- und Sozialrecht in einer Lebenslaufperspektive}

Der Wandel der Strukturen der Erwerbsarbeit wird in den Sozialwissenschaften mit unterschiedlichen Begriffen erfasst. Doch unabhängig davon, ob wir es im Übergang vom Fordismus zum Postfordismus mit Entgrenzung, Subjektivierung, Prekarisierung und/oder Arbeitskraftunternehmertum zu tun haben: Es ist schon fast zu einem Gemeinplatz geworden, dass sich aus diesem Wandel grundsätzliche Herausforderungen für das Arbeits- und Sozialrecht und dessen normative Leitvorstellungen (»Normalarbeitsverhältnis «) ergeben. ${ }^{2}$

Insbesondere die sozialwissenschaftliche Lebenslaufperspektive hat einige dieser Herausforderungen auf den Punkt gebracht. Eine am Lebenslauf orientierte Politik müsse "gleichzeitig darauf [abzielen], ArbeitnehmerInnen beziehungsweise BürgerInnen in die Lage zu versetzen, Erwerbsarbeit und andere sinnvolle Aktivitäten - wie Fürsorgearbeit - zu einem bestimmten Zeitpunkt zu synchronisieren sowie zugleich vielfältige Präferenzen und Entscheidungen über den Lebensverlauf zu verteilen (zu diachronisieren) «. ${ }^{3}$ Eine Neubetrachtung des Arbeits- und Sozialrechts aus der Perspektive der Lebensverläufe und Erwerbskarrieren konzentriert sich auf Situationen der Diskontinuität als Schnittstellen für eine Neuorientierung des Rechts. Übergangssituationen der Erwerbsarbeit werden hierfür aus der Perspektive der Lebensverläufe von Beschäftigten und damit reflexiv in Hinblick auf ihre Konsequenzen ${ }^{4}$ betrachtet. Gerade weil die tatsächliche Betroffenheit der Beschäftigten durch Diskontinuität und Destabilisierung sich je nach Qualifikation, Geschlecht, Alter und Herkunft stark unterscheidet, ${ }^{5}$ muss vom Recht im Wohlfahrtsstaat erwartet werden, dass Entscheidungsfreiheit und Selbstbestimmung auch in Übergangssituationen und bei Unsicherheit gewährleistet sind.

Bei der Suche der EU nach neuen Gesamtkonzepten für die rechtliche Regulierung der Erwerbsarbeit haben die Analysen des Supiot-Berichts großen Widerhall gefunden. Der Rechtswissenschaftler Alain Supiot war von der Europäischen Kommission mit der Leitung einer ländervergleichenden interdisziplinären Studie beauftragt worden, um konzeptionelle Grundlagen für die arbeits- und sozialrechtliche Regulierung der heutigen Europäischen Union zu entwickeln. Er kam zu dem Ergebnis, dass Regulierungen den sozialen Schutz ermöglichen müssten, um mit wachsender Unsicherheit umzugehen, und dabei zu berücksichtigen hätten, dass Beschäftigte im Laufe ihres Erwerbslebens zunehmend mehrere Arbeitstätigkeiten in unterschiedli-

2 Siehe die Fragestellung des 68. Deutschen Juristentags 2010: »Abschied vom Normalarbeitsverhältnis? «; zur Debatte Kocher, Welti 2010; genauer zu den Unterschieden zwischen einer empirischen und einer normativen Auffassung des Begriffs Pfarr 2000; zu den tatsächlichen Veränderungen siehe zum Beispiel Schmid 2012, S. 250 ff.

3 Klammer 2010, S. 699; zu dem Begriff der »Lebenslaufpolitik « auch Leisering 2003; vgl. in Bezug auf die Arbeitszeit auch Wotschack 2011, S. 99 ff.

4 Siehe zu diesem Begriff von »Reflexivität« die Einleitung in Naegele, Bertermann 2010. Zum Konzept des reflexiven Rechts, das sich auf andere Gegenstände der Reflexivität bezieht, siehe Fußnote 63.

5 Siehe zum Beispiel Giesecke, Heisig 2010. 
chen Beschäftigungsformen ausübten. Der Schutz dürfe deshalb nicht zu stark mit dem einzelnen Arbeits- und Beschäftigungsverhältnis verklammert sein, da er über Unterbrechungen und Übergänge hinweg gleich wirksam bleiben müsse. ${ }^{6}$

\subsection{Bürger- und Menschenrechte in der Erwerbsarbeit}

Der Supiot-Bericht schlägt insbesondere vor, die Regulierungsvorschläge für die Erwerbsarbeit in vier Rechtskreisen zu systematisieren: Es seien Regeln zu finden für die abhängige Erwerbsarbeit im engeren Sinn, für die berufliche Arbeit, für die nichtberufliche Arbeit sowie - im äußersten Kreis - für jegliche Tätigkeit unabhängig vom konkreten Beschäftigungsverhältnis. ${ }^{7}$ Spätestens im äußersten Kreis findet dieser Vorschlag Anschluss an Konzepte für fundamentale Grundrechte der Arbeit. ${ }^{8}$ Insbesondere der Fähigkeiten-Ansatz (»capabilities «) $)^{9}$ erscheint als geeigneter normativer Rahmen für ein solches Gesamtkonzept, wenn man die Aufgabe fundamentaler Rechte darin sieht, jedem Menschen die Möglichkeit zu bieten, die eigenen Fähigkeiten und Wünsche - unter Berücksichtigung der tatsächlichen sozialen Bedingungen - zu verwirklichen. ${ }^{10}$

Alain Supiot hat das Gesamtkonzept mit dem Begriff eines neuen "statut professionel « umschrieben. Dieser Begriff wird häufig in der französischen Fassung zitiert, da selbst Supiots eigene Übersetzungsversuche unzulänglich erscheinen (»labour force membership status « in der englischen Fassung ${ }^{11}$ ); auch die mögliche deutsche Übersetzung »Erwerbstätigenstatus « überzeugt nicht vollständig. Solche Übersetzungen leiden unter dem Ballast der mindestens impliziten Bezugnahmen auf die jeweiligen historisch geprägten institutionellen Arrangements und Rechtsordnungen. Es bedarf deshalb nicht nur einer begrifflichen Übersetzung, sondern auch einer auf den Pfad der konkreten Rechtsordnung bezogenen Re- und Ausformulierung. Für die deutsche Debatte erscheint als Ausgangspunkt hierfür der Begriff des »Sozialen Rechts « interessant, der in der Rechtstheorie der Weimarer Republik mit dem Anspruch verbunden war, die realen Machtverhältnisse hinter der formalen Gleichheit der Rechtssubjekte in den Blick zu nehmen und normativ den »Menschen im

6 Supiot 2001.

7 Ebd, S. 50 ff.; daran anschließend Perulli 2003, S. 104 ff.; Europäisches Parlament, Entschließung vom 6. Juli $2010 \mathrm{zu}$ atypischen Verträgen, gesicherten Berufslaufbahnen, Flexicurity und neuen Formen des sozialen Dialogs (2009/2220(INI)), Nr. 6 und 29; vgl. auch Freedland, Kountouris 2011, S. 200: "personal work profile «; zu den Gefahren einer »Überwindung « des Beschäftigungsverhältnisses Castel 2011, S. 106.

8 So auch zum Beispiel Arthurs 2011, S.23; zur Menschenrechtsdebatte in Bezug auf Arbeitsrechte siehe Fudge 2007; Kocher 2012.

9 Sen 2010; Nussbaum 2008.

10 Fudge 2007; Supiot 2009; zur Bedeutung des »capabilities «-Ansatzes siehe auch Schmid 2011, S. 50 ff.; Bonvin 2012; Drouin 2009, S. 152 ff.; Deakin, Rogowski 2011, S. 238 ff.

11 Supiot 2001, S. 50 ff; für eine Rekonstruktion der französischen Debatte siehe auch Castel 2011, S. $100 \mathrm{f}$. 
Recht « in den Mittelpunkt zu stellen. ${ }^{12}$ Im Folgenden soll diskutiert werden, wie die Anforderungen eines lebenslauforientierten Arbeits- und Sozialrechts als Teilaspekte des »Sozialen Rechts der Arbeit « pfadgemäß für das deutsche Recht ausformuliert werden könnten.

\subsection{Ziehungsrechte für Zeit und Bildung}

Hierfür scheint auf den ersten Blick beispielsweise der Begriff der Ziehungsrechte (»social drawing rights «) geeignet. ${ }^{13} \mathrm{Im}$ Rahmen des von Supiot entwickelten Konzepts bezeichnet er Rechte, die dazu beitragen sollen, Unterbrechungen und Übergänge im Arbeitsleben ohne Angst vor Unsicherheit zu ermöglichen und Mobilität beziehungsweise Flexibilität und Sicherheit für die Beschäftigten miteinander zu versöhnen. ${ }^{14}$ Der Begriff wird in der Regel einerseits im Zusammenhang mit Rechten und »Optionen « auf Zeit verwendet, ${ }^{15}$ andererseits im Zusammenhang mit dem Recht auf (lebenslange) Weiterbildung. ${ }^{16}$

Vergleichbare Grundanliegen finden sich in der europäischen Debatte um die Verbindung von Flexibilität und Sicherheit (»Flexicurity «) wieder, hier unter stärker arbeitsmarktpolitischen Vorzeichen. So war das arbeitsrechtliche Grünbuch der Europäischen Kommission, zu dessen Vorbereitung die Arbeiten von Supiot und anderen gedient hatten, ${ }^{17}$ zwar an seinem deregulierenden Impetus gescheitert. Seine konzeptionellen Anliegen wurden jedoch nicht aufgegeben, sondern führten zu Forderungen nach Integration destandardisierter Erwerbsbiografien, nach "garantierten Optionalitäten « der Zeitsouveränität einschließlich der Absicherung von Zeiten ohne Erwerbsarbeit, ${ }^{18}$ nach Rechten auf lebenslanges Lernen sowie Arbeitsmarktübergängen und internen Flexibilitäten. ${ }^{19}$

Der Begriff der Ziehungsrechte, der in diesem Zusammenhang eine wichtige Idee für die Umsetzung darstellt, vereint zwei wichtige Aspekte: ${ }^{20}$ Zum einen betont er die individuelle subjektive Freiheit bei der Wahrnehmung zum Beispiel von Auszeiten oder Weiterbildungsmöglichkeiten. Zum anderen will er Klarheit hinsichtlich der zur Verfügung stehenden Ressourcen ermöglichen: Jeder und jede Beschäftigte

12 Eichenhofer 2012; Seifert 2011; aus der damaligen Zeit siehe vor allem Radbruch 1957.

13 Supiot 2001, S. $56 \mathrm{f}$.

14 Mückenberger 2007, S. 199; Castel 2011, S. 146.

15 Mückenberger 2007, S. 196: »zeitbezogene Optionsrechte von Beschäftigten mit Blick auf die für ihre Lebenslagen und biographischen Verläufe spezifischen Zeitbedarfe « (dort auch zur Herkunft aus der internationalen Währungspolitik); Sachverständigenkommission 2006, S. 266 ff.: »Optionszeiten «; vgl. auch Wotschack 2011, S. 99 ff.

16 Schmid 2011, S. 98 ff.; Schmid 2012, S. 216 ff.; vgl. auch Castel 2011, S. 102.

17 Europäische Kommission 2006; für den Flexicurity-Begriff insbesondere Wilthagen, Tros 2004.

18 Klammer 2005, S. 260 ff.

19 Europäische Kommission 2007; siehe jetzt Europäische Kommission 2012; Klammer 2005; Blanke, Bleses 2005.

20 Supiot 2001, S. 56. 
soll danach aus einer individuell garantierten Reserve schöpfen können. Hierfür seien »accounts, credits, savings, vouchers « erforderlich, die durch Staat, Sozialversicherung, Unternehmen, Verbände oder Arbeitnehmer geschaffen werden könnten - abhängig vom Nutznießer der Ziehung. ${ }^{21}$

\subsection{Kontenmodelle und das Problem der Sichtbarmachung von Verteilungsfragen}

Als Rahmen für die Schaffung solcher "accounts, credits, savings, vouchers " in Form individuell garantierter Reserven sind Konten und Fonds vorgeschlagen worden. ${ }^{22}$ Diese Modelle verwirklichen beide Aspekte - individuelle Freiheit und garantierte Reserve -, da individuelle (wenn auch meist begrenzte) Ressourcen garantiert werden, sodass Anträge und Wünsche keiner Prüfung auf Notwendigkeit und »Förderungswürdigkeit « mehr unterzogen werden müssen. Jede selbstbestimmte Entscheidung wird als legitim anerkannt und gefördert; es entstehen Anreize auch für riskante Entscheidungen. ${ }^{23}$

Schon das Ziel, Menschen zu dem Zeitpunkt im Leben Ressourcen zur Verfügung zu stellen, zu dem sie diese benötigen, ${ }^{24}$ kann dabei aber leicht verfehlt werden: In der mittleren Lebensphase zwischen Erwerbseinstieg und Ende der Kindererziehung (»rush hour " des Lebens), in der die meisten Ressourcen benötigt werden, sind häufig noch nicht ausreichend Ziehungsrechte angespart. ${ }^{25}$ Andererseits mag es auch den Fall geben, dass die Erkenntnis der » richtigen « Zeit- oder Bildungsnutzung individuell erst reift, nachdem das Recht bereits gezogen wurde. Die Ansparlogik wird in den vorliegenden Modellen zur Weiterbildung deshalb zu Recht eingeschränkt. Die Summe müsse nicht individuell angespart worden sein, und die Rechte sollen mehrfach und über die insgesamt anzusparende Summe hinaus in Anspruch genommen werden können. ${ }^{26}$

Ein weiteres Problem von Konten betrifft die Frage nach der Gerechtigkeit in der Verteilung gesellschaftlicher Ressourcen: Wenn für jede Person dieselbe Summe zur Verfügung gestellt wird, kann nicht mehr nach unterschiedlichen Bedürfnissen differenziert werden (beispielsweise Bedürfnisse von Personen mit unzureichender oder verfallender Qualifikation, Personen mit einer Kumulation riskanter Übergänge

21 Ebd., S. 57; Mückenberger 2007, S. 200.

22 Siehe den Überblick bei Käpplinger et al. 2013, S. 58 ff.; Schmid 2011, S. 98 ff.: »Persönliches Entwicklungskonto" (so auch Hipp 2009, S. 366 ff.); Schmid 2012, S. 216 ff.: »Individuelle Ziehungsrechte aus einem solidarischen Weiterbildungsfonds «; vgl. auch Expertenkommission Finanzierung Lebenslangen Lernens 2004, S. 188: „Bildungssparen «.

23 Schmid 2011, S. 99; S. 160 f. auf der Basis einer verhaltenstheoretischen Analyse; siehe auch Sachverständigenkommission 2006, S. 267 ff. mit einer geschlechterpolitischen Begründung.

24 Schmid 2012, S. 263.

25 Mückenberger 2007, S. 198.

26 Schmid 2012, S. 263; vgl. auch Bosch 2010, S. 39: »Beschäftigungslogik « statt Ansparlogik.

Leviathan, 41. Jg., 3/2013 
oder einer Vielzahl von Sorgepflichten). ${ }^{27}$ Es werden also mit der Vergabe dieser Ziehungsrechte (unsichtbare) Verteilungsentscheidungen getroffen. Die "richtige " Entscheidung für die »richtige « Weiterbildung zum » richtigen « Zeitpunkt erfordert aber ohnehin bereits hohe Bildungskompetenz; dies ist vermutlich einer der Gründe dafür, dass diejenigen Personen mit dem höchsten Bildungsbedarf am seltensten Weiterbildung in Anspruch nehmen. ${ }^{28}$

Schränkt man die Ziehung aus einem Konto jedoch durch bedarfsorientierte Kriterien ein, befindet man sich auf dem Weg in ein Versicherungsmodell, das die Frage nach der richtigen Verteilung gesellschaftlicher Ressourcen anders löst als durch individuell garantierte Summen: Die Ressourcen würden dann nicht in der Höhe, sondern in der Sache begrenzt. Durch die Benennung konkreter Anspruchsvoraussetzungen müssen dann Kriterien für die finanzielle Förderungswürdigkeit von Auszeiten und/oder Weiterbildungsmaßnahmen gefunden werden, die einer sozialpolitisch gerechten Verteilung öffentlicher und gesellschaftlicher Ressourcen entsprechen.

Dies ist überall dort sachgerecht, wo der Staat (oder die Versichertengemeinschaft beziehungsweise der mit öffentlichen Mitteln ausgestattete Fonds) Ressourcen zur Verfügung stellt. Denn die öffentliche Hand sollte die finanziellen und sonstigen sozialen Risiken einer individuellen Entscheidung nur tragen, wo diese Entscheidung aus gesellschaftlicher Sicht sinnvoll und förderungswürdig erscheint. Unternehmen und Arbeitgeber wiederum sollten hingegen finanzielle Lasten dort übernehmen, wo sie die sozialpolitische Verantwortung tragen oder von den Vorteilen profitieren (wie insbesondere im Fall der betrieblichen Weiterbildung ${ }^{29}$ ).

Die Entnahme beziehungsweise die Inanspruchnahme der Ressourcen und die Einhaltung von Kriterien müssten dann allerdings durch (sozialversicherungsrechtliche, behördliche oder andere) Entscheidungsträger und durch rechtlich ausgestaltete Verfahren kontrolliert werden.

\subsection{Das Recht auf eine selbstbestimmte Erwerbsbiografie (Artikel 12 GG)}

Eine Begrenzung von Leistungen durch Sachkriterien erscheint deshalb vorzugswürdig gegenüber einer bedingungslosen Ziehung. Diese Begrenzung bei gleichzeitiger Autonomie können differenzierte Rechtsansprüche leisten. Das Ziel der Selbstbestimmung in sozialer Einbindung, das mit solchen Ansprüchen verfolgt werden sollte, lässt sich insofern für die deutsche Rechtsordnung im verfassungsrechtlichen Rahmen eines »Rechts auf eine selbstbestimmte Erwerbsbiografie « ausformulieren. ${ }^{30}$

Ein solches Recht ergibt sich im Grundsatz bereits aus dem Grundgesetz. Die Berufsfreiheit (Artikel 12 GG) enthält Rechte auf berufliche Entwicklung und Wei-

27 Welti et al. 2012, S. 187.

28 Kruppe 2013.

29 Vgl. die Vorschläge für Handlungsanreize im Rahmen der Finanzierung (»levyexemption-System «); Bosch 2010, S. 7 f.

30 So auch der Titel des Buchs mit den Ergebnissen des Projekts SozRA (Kocher et al. 2013). 
terentwicklung auch für abhängig beschäftigte Arbeitnehmerinnen und Arbeitnehmer. ${ }^{31}$ Darüber hinaus gewährleisten die Grundrechte auf Privat- und Familienleben eine angemessene Abstimmung der Erwerbsarbeit mit sogenannten privaten Anliegen. Die Integration von behinderten und gesundheitlich eingeschränkten Menschen ins Erwerbsleben, die Vereinbarkeit von nichterwerbsmäßiger Sorgearbeit, Familie und Beruf sowie die berufliche Fort- und Weiterbildung sind durch spezielle Rechte auf Gleichbehandlung, Förderung, angemessene Vorkehrungen und Persönlichkeitsentwicklung verfassungsrechtlich abgesichert. Das Recht auf eine selbstbestimmte Erwerbsbiografie hat also auch zum Gegenstand, Entscheidungen und Lebensläufe, die von der männlichen sozialen Norm abweichen, rechtlich zu akzeptieren - und Übergangssituationen entsprechend rechtlich zu gestalten. Die Beschäftigten müssen in der Erwerbsarbeit selbst mit darüber entscheiden können, wie persönliche und organisatorische Konflikte gelöst werden, anstatt ohne Not Normalitätsvorstellungen und Normalisierungszwängen unterworfen zu werden.

Dieses Recht und die Überlegungen, die mit Ziehungsrechten meist verbunden sind, verfolgen also ähnliche Ziele. Der Begriff »Ziehungsrecht « stellt allerdings die Technik der Finanzierung von Ansprüchen in den Mittelpunkt und erschwert die Sichtbarmachung von Verteilungsentscheidungen, indem er Kontenmodelle nahelegt. Das Recht auf eine selbstbestimmte Erwerbsbiografie bezieht sich demgegenüber auf den verfassungsrechtlichen Kontext des deutschen Rechts, ist also normativ gesetzt und benennt unmittelbar die geschützten Interessen an einer selbstbestimmten Erwerbsbiografie.

Das Recht auf eine selbstbestimmte Erwerbsbiografie regelt einen Ausschnitt aus dem Gesamtkomplex fundamentaler Rechte der Erwerbsarbeit und benennt die normative Zielsetzung, der eine lebenslauforientierte arbeits- und sozialrechtliche Regulierung zu folgen hätte. Es steht allerdings gleichzeitig für eine Begrenzung individueller Leistungen durch Sachkriterien, die demokratisch festzulegen und zu legitimieren wären - und deren Einhaltung durch Entscheidungsträger kontrolliert werden müsste. Für die Entscheidungen über die Gewährung dieser (Ziehungs-)Rechte bedarf es also rechtlich gestalteter Verfahren. Soll die erforderliche Selbstbestimmung nicht an dieser Stelle verloren gehen, ist eine Verfahrensform nötig, welche entstehende Konflikte angemessen zu lösen in der Lage sein müsste. Man kann über Recht nicht reden, ohne gleichzeitig Verfahren zu betrachten.

In Bezug auf das Recht auf eine selbstbestimmte Erwerbsbiografie geht es dabei um Verfahren auf mindestens zwei Ebenen, denn zur Verwirklichung des Rechts bedarf es der Durchsetzung von Ansprüchen auf der betrieblichen Ebene (im Verhältnis zum Arbeitgeber, eventuell mit Unterstützung des Betriebsrats) und auf der Ebene der Behörden (im Verhältnis zum Staat einschließlich der Gemeinden oder Sozialversicherungsträger). Welche Ansprüche hier mit welchen Verfahren für die betriebliche Ebene und für die Finanzierungsfragen verbunden werden könnten, soll im Folgenden skizziert werden.

31 BVerfG 11.6.1958, BVerfGE 7, 377, 397; BVerfG 15.12.1987, BVerfGE 77, 308, Rz. 98 (Weiterbildungsgesetze); BVerfG 25.1.2011, BVerfGE 128, 157 ff.; Bryde 1984; Schneider 1985. 


\section{Ansprüche zur Verwirklichung des Rechts auf eine selbstbestimmte Erwerbsbiografie}

\subsection{Subjektive Rechte und Ansprüche}

Geht es um die Umsetzung von Rechten in konkrete Regelungen, so bedarf es einer Differenzierung der einzelnen Ansprüche. So ist die Verwirklichung von Rechten auf »Zeit « (das heißt auf Auszeit, Arbeitsreduzierung, Neufestlegung von Arbeitszeiten, Arbeitszeitaufstockung) genau wie zum Beispiel die Verwirklichung von Rechten auf Weiterbildung auf eine ganze Reihe von Ressourcen angewiesen: Zeitsouveränität; Finanzierung des Einkommensersatzes beziehungsweise der Kosten des Lebensunterhalts; Finanzierung von Dienstleistungen wie der Qualifizierungsmaßnahme oder Finanzierung einer Anpassung des Arbeitsplatzes (etwa gesundheitsangepasste Arbeitsmittel); Finanzierung sekundärer Risiken wie Krankheit oder Alterssicherung/Rente ${ }^{32}$. Für all diese Ressourcen kommen jeweils unterschiedliche Verteilungsentscheidungen und Entscheidungsverfahren und damit unterschiedliche Ansprüche in Betracht.

In der Rechtstheorie wird zwischen »subjektivem Recht « und »Anspruch « unterschieden. Während das subjektive Recht den rechtlichen Schutz eines bestimmten Interesses (hier: der selbstbestimmten Erwerbsbiografie) bezeichnet, handelt es sich beim Anspruch um ein Forderungsrecht ( $\mathbb{1} 194$ BGB), das heißt um die konkrete einklagbare Forderung gegen eine andere (juristische oder natürliche) Person beziehungsweise Institution (hier: den Anspruch auf Arbeitszeitreduzierung gegen den Arbeitgeber oder den Anspruch auf Finanzierung des Lebensunterhalts gegen die Sozialversicherung). Jedes subjektive Recht bedarf zu seiner Verwirklichung der Benennung von mehreren Ansprüchen gegen bestimmte Institutionen und Personen.

\subsection{Das geltende Recht}

Das subjektive Recht auf eine selbstbestimmte Erwerbsbiografie kann also nur durch konkrete Einzelansprüche gegenüber Arbeitgeber und Sozialversicherungsträger oder anderen Institutionen rechtlich Anerkennung finden. Die hierfür erforderlichen zahlreichen konkreten Ansprüche in unterschiedlichen Kontexten können an dieser Stelle nicht im Einzelnen dargestellt werden. Auch die geltende Rechtslage ist komplex, disparat und in einem ständigen Wandel begriffen. Die Ausgestaltung der Rechte für Mobilitätssituationen »ist kein Patentrezept, sondern eher eine Baustelle. « ${ }^{33}$ Zur Veranschaulichung sollen im Folgenden exemplarisch einige wesentliche Elemente herausgegriffen werden, für die es bereits Regelungen im geltenden Recht gibt. Es handelt sich dabei um Rechte auf Anpassung des Erwerbsarbeitsverhältnisses an die individuellen lebenslaufbezogenen Erfordernisse, insbesondere Rechte auf Zeitsouveränität und Weiterbildung sowie um die Finanzierung solcher Übergänge.

32 Nur auf diesen letzten Aspekt bezieht sich das Optionszeitenmodell des Siebten Familienberichts der Bundesregierung; siehe Sachverständigenkommission 2006, S. 266 ff.

33 Castel 2011, S. 213. 


\section{Das Recht auf individuelle Anpassung von Arbeitsverhältnissen}

Für die Anpassung von Arbeitsverhältnissen an individuelle Bedürfnisse gibt es nach geltendem Recht ${ }^{34}$ in bestimmten Fällen gesetzliche Ansprüche auf erwerbsarbeitsfreie Zeit zu dem jeweils erforderlichen Zeitpunkt (zum Beispiel bei der Schwangerschaft ${ }^{35}$, bei Elternzeit ${ }^{36}$, Pflegezeit ${ }^{37}$ oder bei Krankheit ${ }^{38}$ ). Darüber hinaus existieren Rechtsgrundlagen für die Reduzierung der Arbeitszeit ${ }^{39}$ sowie für den Wechsel von der Nachtarbeit auf einen Tagesarbeitsplatz bei Betreuung von Kindern oder gesundheitlichen Problemen. ${ }^{40}$ Auch für (Schwer-)Behinderte gibt es einen Anspruch auf variable Gestaltung der Arbeitszeiten. ${ }^{41}$ Aus allgemeinen zivil- und arbeitsrechtlichen Vorschriften lassen sich darüber hinaus weitergehende Ansprüche auf Anpassung an unterschiedliche individuelle Bedürfnisse entnehmen. So können sich Ansprüche der Beschäftigten auf Anpassungsmaßnahmen sowohl aus der »Fürsorge «-Pflicht des Arbeitgebers ${ }^{42}$ ergeben als auch aus den (verfassungsrechtlichen) Grenzen des Weisungsrechts nach $\$ 106$ der Gewerbeordnung (GewO) oder der Arbeitgeberpflicht zur Prävention von Diskriminierung ${ }^{43}$. Bei einer sachgerechten Auslegung der meisten Arbeitsverträge sind nach geltendem Recht in aller Regel auch arbeitsrechtliche Ansprüche auf Freistellung für eine betriebsnotwendige Weiterbildung herzuleiten (soweit dies angesichts des allgemeinen Teilzeitanspruchs noch erforderlich erscheint). ${ }^{44}$

Arbeitsrechtliche Ansprüche zur Verwirklichung des Rechts auf selbstbestimmte Erwerbsbiografien müssen also nicht neu erfunden werden. Einen allgemeinen Anpassungsanspruch gesetzlich vorzugeben wäre aber eine Erleichterung und würde zur Transparenz beitragen; die bestehenden Ansprüche könnten dann systematisch

34 Siehe zu vergleichbaren Entwicklungen im Recht des Vereinigten Königreichs und im USamerikanischen Recht auch Durkalski 2009, S. 385 ff.

$35 \mathbb{S} 3 \mathrm{ff}$. MuSchG (Mutterschutzgesetz).

$36 \mathbb{S} \mathbb{S} 15 \mathrm{ff}$. BEEG (Bundeselterngeld- und Elternzeitgesetz).

37 \\$ 2 ff. PflZG (Pflegezeitgesetz von 2008).

38 \275 BGB (Bürgerliches Gesetzbuch); das Entgeltfortzahlungsgesetz (EntgeltFZG) regelt demgegenüber nur die Frage des Entgelts in dieser Zeit.

39 Während der Elternzeit nach BEEG, während einer Pflegezeit nach dem PflZG, als allgemeiner »Teilzeitanspruch « in $\mathbb{8} 8 \mathrm{TzBfG}$ (Teilzeit- und Befristungsgesetz). Das Familienpflegezeitgesetz (FamPflZG) von 2012 enthält dagegen lediglich Rechte auf Förderung von Arbeitszeitkonten, jedoch keine eigenständigen Anpassungs- oder Arbeitszeitansprüche.

$40 \$ 6$ Abs. 4 ArbZG (Arbeitszeitgesetz).

41 \81 Abs. 5 Satz 3 des Neunten Buchs des Sozialgesetzbuchs (SGB IX) für Schwerbehinderte; gleiches gilt bei richtiger Auslegung aber für alle behinderten Arbeitnehmerinnen und Arbeitnehmer (genauer Nassibi 2012, S. 721).

$42 \int 618$ BGB.

$43 \rrbracket 12$ Abs. 1 AGG (Allgemeines Gleichbehandlungsgesetz).

44 Käufer 2002; Kocher, Welti 2013, S. 17 ff.; siehe auch Natzel 2010, S. 700 ff.; zur Finanzierung von Anpassungen siehe weiter unten bei Fußnoten 64 ff.

Leviathan, 41. Jg., 3/2013 
in Hinblick auf dieses Recht begriffen und entsprechend verbessert werden. So fehlt es aktuell etwa an explizit und transparent geregelten Ansprüchen auf befristete Teilzeit oder auf Vollzeit. ${ }^{45}$

\section{»Übergangsmanagement «: Reflexives Recht im betrieblichen Konflikt}

Die erwähnten Rechtsansprüche sehen allerdings häufig vor, dass der Arbeitgeber dem Anpassungswunsch des Arbeitnehmers oder der Arbeitnehmerin »betriebliche Gründe ", »dringende betriebliche/dienstliche Belange " oder »Unzumutbarkeit « entgegensetzen kann. »Betriebliche Gründe « in diesem Sinne liegen vor, wenn sie sich zwingend aus der Arbeitsorganisation oder dem Arbeitsablauf ergeben oder sich aus unverhältnismäßigen Kosten des Arbeitgebers begründen lassen (vgl. $\mathbb{\$} 8$ Abs. 4 TzBfG). Betriebliche Gründe können sich auch aus der Unvereinbarkeit mit den legitimen Zeitinteressen anderer Beschäftigter ergeben. ${ }^{46}$

Die Begrenzung von Anpassungsansprüchen durch solche Einwände ist in der Sache durchaus sinnvoll. Schließlich sind in der Belegschaft oft sich widersprechende Zeitprioritäten gegeneinander abzuwägen und mit der betrieblichen Organisation abzustimmen. ${ }^{47}$ Hier liegen notwendige Grenzen der individuellen Selbstbestimmung, die sich aus der kollektiven und betrieblichen Einbindung der Erwerbsarbeit ergeben.

Da es sich bei den Einwänden im Rahmen von Rechtsansprüchen um rechtliche Einwände handelt, ist gerichtlich zumindest teilweise zu überprüfen, ob die geltend gemachten betrieblichen Interessen tatsächlich vorliegen. ${ }^{48}$ Insofern gewährleistet die Rechtsform größere Rechtssicherheit und Rechtsmacht im Konflikt, als wenn die Abstimmung der Interessen allein der beiderseitigen Willkür überlassen bliebe. Dennoch lässt der Vorbehalt der betrieblichen Interessen Entscheidungsspielräume. ${ }^{49}$ » Wir müssen zwischen der Unbedingtheit des Rechts und den Bedingungen seiner Verwirklichung unterscheiden «: $:{ }^{50}$ Das zentrale Problem bei den Ansprüchen auf die individuelle Anpassung des Arbeitsverhältnisses ist heute nicht mehr die Gewährung eines unbedingten Anspruchs als solchem; die zentrale Frage ist, wie dieser im Konfliktfall auf Ebene des Betriebs zur Wirkung gebracht werden kann.

Der deutsche Gesetzgeber gibt immerhin einige Hinweise darauf, wie er sich dies vorstellen könnte. Denn wir finden im geltenden Recht zunehmend Regelungen über Organisationspflichten der Arbeitgeber ${ }^{51}$ oder - im Rahmen von Arbeitszeitredu-

45 Ausführlich Kocher et al. 2013, S. 162 f.

46 Kocher 2007.

47 Vgl. auch Mückenberger 2007, S. 198.

$48 \mathrm{Zu}$ den Grenzen der gerichtlichen Überprüfung einer »unternehmerischen Entscheidung « ausführlich Kocher 2007.

49 Bundesarbeitsgericht, Urteil vom 16.12.2008, Az. 9 AZR 893/072008.

50 Castel 2011, S. 213.

51 \ 12 AGG. 
zierungsansprüchen - Erfordernisse individueller »Verhandlungen «.52 Bei der behindertengerechten Gestaltung von Arbeitsbedingungen schreibt das Gesetz ${ }^{53}$ mit dem Betrieblichen Eingliederungsmanagement (BEM) ein interessantes Verfahren vor, in dem unter Beteiligung aller betrieblichen Interessenvertretungen sowie mit Hilfe der zuständigen Sozialversicherungsträger ein »offener Suchprozess « und Dialog gestartet werden muss, sobald eine Arbeitnehmerin oder ein Arbeitnehmer Arbeitsunfähigkeitszeiten von mindestens sechs Wochen innerhalb eines Jahres aufweist. ${ }^{54}$ In Hinblick auf die Weiterbildung heißt es im Betriebsverfassungsgesetz, ${ }^{55}$ dass der Arbeitgeber mit dem Arbeitnehmer erörtern muss, "wie dessen berufliche Kenntnisse und Fähigkeiten im Rahmen der betrieblichen Möglichkeiten den künftigen Anforderungen angepasst werden können «, sobald feststeht, dass aufgrund betrieblicher Veränderungen eine solche Anpassung erforderlich wird. Ein systematisches Verfahrensmodell lässt sich zwar noch nicht erkennen. Auch gibt es kaum empirische Daten über die Verwirklichung dieser Verhandlungsverfahren. Allerdings geben die vorliegenden Daten Hinweise darauf, dass eine Einbindung der Interessenvertretungen und externer Akteure, wie sie beim BEM praktiziert wird, größere Verwirklichungschancen verspricht als individuelle Verhandlungen zwischen Arbeitgeber/in und Arbeitnehmer/in. ${ }^{56}$

Insbesondere die betriebliche Mitbestimmung ist für die tatsächliche Verwirklichung solcher Verfahren von nicht zu unterschätzender Bedeutung. Ohne entsprechende Rechte der Interessenvertretungen können die Beschäftigten in individuellen Verhandlungen wenig ausrichten. Nur die regelmäßige Einbeziehung der betrieblichen Interessenvertretungen kann auch Lernprozesse in den Betriebsräten und in der Belegschaft fördern und zur Auseinandersetzung mit Gerechtigkeitsfragen bei der Veränderung von Normalitätsvorstellungen und veränderten privaten Interessen beitragen. Das geltende Recht sieht bislang eine systematische Beteiligung von Betriebsräten allerdings nur für den Fall vor, dass ein Arbeitgeber selbst regelhafte Verfahren zur Behandlung individueller Ansprüche innerbetrieblich institutionalisiert; das Mitbestimmungsrecht ${ }^{57}$ bezieht sich in diesen Fällen auf die Ausgestaltung solcher Verfahren. ${ }^{58}$

$52 \mathbb{S} 8$ Abs. 3 TzBfG, $\mathbb{S} 15$ Abs. 5 Satz 2 BEEG; siehe zu diesen Normen schon Kocher 2007.

$53 \mathbb{S} 84$ Abs. 2 SGB IX - Rehabilitation und Teilhabe behinderter Menschen.

54 Bundesarbeitsgericht, Urteil vom 10.12.2009, Az. 2 AZR 198/09; Kohte 2010 a.

$55 \mathbb{S} 81$ Abs. 4 Satz 2 BetrVG (Betriebsverfassungsgesetz).

56 Zum BEM siehe zum Beispiel Niehaus, Vater 2010, S. 194 ff.; zu den entsprechenden Daten aus der WSI-Betriebsräte-Sonderbefragung 2011 siehe Kocher et al. 2013, S. $134 \mathrm{ff}$.

57 Einschlägig ist die Mitbestimmung über Fragen der Ordnung des Betriebs und des Verhaltens der Arbeitnehmer im Betrieb ( $\mathbb{S} 87$ Abs. 1 Nr. 1 BetrVG).

58 Zum Mitbestimmungsrecht nach $\mathbb{\int} 87$ Abs. 1 Nr. 1 BetrVG bei der Einrichtung von Beschwerdestellen nach dem Allgemeinen Gleichbehandlungsgesetz ( $\mathbb{1 3}$ AGG) siehe Bundesarbeitsgericht, Urteil vom 21.07.2009, Az. 1 ABR 42/08; zur Verallgemeinerung siehe Kocher et al. 2013, S. 308 f. 
Zur Bearbeitung der mit individuellen Anpassungswünschen verbundenen Abstimmungsfragen bietet es sich einerseits an, nach den bislang verstreuten Vorbildern im geltenden Recht, insbesondere nach dem Vorbild des BEM, weitere Verfahren des »betrieblichen Übergangsmanagements " einzuführen, in denen die Pflicht zur Einleitung »offener Suchprozesse « zu bestimmten Eingreifzeitpunkten vorgesehen ist, zum Beispiel nach längeren Auszeiten (insbesondere bei der Rückkehr aus der Elternzeit ${ }^{59}$ ) oder nach längerer unterwertiger Beschäftigung. Ein gesetzliches »betriebliches Qualifizierungsmanagement « in Anknüpfung an die erwähnte Erörterungspflicht des Betriebsverfassungsgesetzes ${ }^{60}$ könnte sich an tariflichen Vorbildern orientieren, die überbetriebliche Agenturen und Beratungseinrichtungen in die Rechtsdurchsetzung einbinden. ${ }^{61}$ Die Betriebsräte wären jeweils generell in die individuellen Verhandlungen bei Anpassungsansprüchen einzubeziehen (hierfür müssten insbesondere Mitbestimmungsrechte über den »Regelungsspielraum « der » betrieblichen Interessen « vorgesehen werden). ${ }^{62}$

Solche Verfahren konfrontieren die betrieblichen Logiken und Interessen sowohl mit individuellen als auch mit kollektiven Arbeitnehmerinteressen. Dies ist eine angemessene Form des Umgangs mit der funktionalen Ausdifferenzierung gesellschaftlicher Systembereiche. Denn diese hat zur Folge, dass der Bereich der Erwerbsarbeit aufgrund seiner Eigendynamik als Teil des Wirtschaftssystems nur begrenzt durch materiale rechtliche Vorgaben regulierbar ist. Das Recht muss also » reflexiv « werden, das heißt es muss selbstreflexive Selbstregulierung anderer Systeme dadurch fördern, dass es prozedurale Vorgaben macht ${ }^{63}$ - und genau dies können die hier vorgestellten Verfahren leisten.

\section{Finanzierung von Anpassungen}

Wenn das Recht auf eine selbstbestimmte Erwerbsbiografie wirksam durchsetzbar werden soll, bedarf es neben den soeben diskutierten Ansprüchen auf Anpassung des Arbeitsverhältnisses und den dazugehörenden Verfahrensvorschriften eines zweiten Bündels von Ansprüchen, um die Fragen der Finanzierung zu regeln. Die Problematik der Finanzierung von Anpassungsrechten lässt sich an dieser Stelle leider nicht einmal ansatzweise in der notwendigen Differenziertheit darstellen. So wäre zwischen unterschiedlichen Kosten (Lebensunterhalt, sekundäre soziale Risi-

$59 \mathrm{Zu}$ den Anforderungen des europäischen Rechts insofern siehe etwa Kohte $2010 \mathrm{~b}$.

60 \81 Abs. 4 Satz 2 BetrVG.

61 Zur möglichen Einbindung kommunaler Ämter für Ausbildungsförderung siehe auch Schmid 2012, S. 263.

62 Zum Vorbild des $\mathbb{8} 87$ Abs. 1 Nr. 5 BetrVG siehe schon Kocher 2007; zum geltenden Recht am Beispiel der Arbeitszeit Paschke 2012; genauer zum Ganzen Kocher et al. 2013, S. $307 \mathrm{f}$.

$63 \mathrm{Zu}$ diesem (aus der systemtheoretischen Rechtslehre stammenden) Begriff des reflexiven Rechts grundlegend Teubner, Willke 1984; siehe auch Deakin, Rogowski 2011, S. 230 ff.; in Bezug auf die hier anstehenden Fragen Kocher 2009, S. 8 ; 34 ff. 
ken, ${ }^{64}$ Kosten der Übergangsmaßnahme selbst) zu unterscheiden; die Kostenübernahme ist auch zwischen möglichen Finanzierungsträgern angemessen aufzuteilen. Einige Beispiele sollen aber zeigen, in welchen Bereichen hier Regelungen zu treffen sind und welche Probleme das geltende Recht aufweist.

So gibt es zwar für die Elternzeit eine aus Steuermitteln finanzierte Entgeltersatzleistung, ${ }^{65}$ für den Fall, dass die Beschäftigen vorübergehend die Pflege von Angehörigen übernehmen, existiert jedoch kein entsprechender Anspruch auf Entgeltersatz. In Situationen einer gesundheitlich bedingten Reduzierung der Arbeitszeit steht eine Teilsicherung nur sehr eingeschränkt zur Verfügung, es fehlt an Kongruenz zum Recht der (Teil-)Erwerbsminderungsrente. Die Kosten in diesen und anderen Übergangssituationen sind je nach Situation und Kontext teils von der Sozialversicherung, teils vom Staat, teils vom Arbeitgeber und teils von den Beschäftigten selbst zu tragen. ${ }^{66}$

In den letzten Jahren ist insbesondere die Frage der Finanzierung von Weiterbildung lebhaft diskutiert worden. ${ }^{67}$ Auch hier zeigt sich die Breite und Differenziertheit der Regelungsnotwendigkeiten. Aktuell bestehen für die Finanzierung von Weiterbildungsrechten arbeitsrechtliche Ansprüche in Hinblick auf die betriebsnotwendige Weiterbildung, die sich nicht nur auf Freistellung, sondern auch auf Finanzierung des Lebensunterhalts, der sekundären sozialen Sicherung sowie der Maßnahme selbst durch den Arbeitgeber beziehen. ${ }^{68}$ Weitere Ansprüche auf Finanzierung von Qualifizierungsmaßnahmen sowie von Entgeltersatz durch die Sozialversicherung sind in $\mathbb{S} \mathbb{S} 81 \mathrm{ff}$. SGB III geregelt; sie erfassen unter bestimmten Voraussetzungen bereits heute die Förderung der individuellen Beschäftigungsfähigkeit.

Die Erfahrungen mit der Finanzierung durch die Agenturen für Arbeit und insbesondere mit den dortigen Entscheidungsverfahren sind jedoch vorsichtig ausgedrückt nicht durchweg positiv, weder in Hinblick auf individuelle Bedürfnisse noch auf arbeitsmarktpolitische Anforderungen. Dies dürfte eine wichtige Motivation für die Suche nach Regelungsmodellen sein, die stärker die Entscheidungsfreiheit des Einzelnen betonen. Die Vorschläge für eine Beschäftigungs- beziehungsweise Arbeitsversicherung schlagen dennoch eine Verallgemeinerung der sozialversiche-

64 So bezieht sich wohl das Optionszeitenmodell des Siebten Familienberichts der Bundesregierung allein auf die Finanzierung des Alterssicherungsrisikos (Sachverständigenkommission 2006, S. 266 ff.).

$65 \mathbb{} 2$ BEEG.

66 Zum Ganzen Kocher et al. 2013, S. 314 ff.

67 Siehe etwa Expertenkommission Finanzierung Lebenslangen Lernens 2004; Bosch 2010, S. 40 f.; Schmid 2011; Kruppe 2013.

68 Käufer 2002; Natzel 2010, S. 700 f.; Kocher, Welti 2013, S. 19 ff.; vgl. SPD-Regierungsprogramm 2013-2017, S. 23. 
rungsrechtlichen Unterstützung jedenfalls hinsichtlich der Förderung (und des Erhalts) der individuellen Beschäftigungsfähigkeit vor. ${ }^{69}$

\subsection{Zur methodischen Trennung der Ansprüche auf Anpassung und auf Finanzierung}

Hier wird also eine Trennung zwischen den Ansprüchen auf arbeitsvertragsrechtliche Anpassung (die sich gegen den Arbeitgeber richten müssen) und den rechtlichen Ansprüchen auf Finanzierung von Kosten und sozialen Risiken (für die unterschiedliche Finanzierungsträger in Betracht kommen) vorgeschlagen. Diese Trennung erscheint sinnvoll, um differenzierte Regelungen treffen zu können. Was das im Einzelnen bedeuten kann, soll im Folgenden beispielhaft gezeigt werden.

\section{Unterschiedliche Voraussetzungen für Anpassung und Finanzierung}

So ist es zunächst keineswegs erforderlich, etwa als Voraussetzung der Wahrnehmung eines sozialversicherungsrechtlichen Anspruchs auf Finanzierung eine Abstimmung mit der Betriebsleitung vorzusehen. Zwar können Ansprüche auf finanzielle Unterstützung nur wirksam werden, wenn es flankierende Ansprüche auf Anpassung (beispielsweise der Arbeitszeit) gibt, die gegen den Arbeitgeber gerichtet werden können. Dies bedeutet jedoch nicht, dass die Anspruchsvoraussetzungen miteinander verknüpft werden müssen. Die Trennung ermöglicht es, für die Anpassungsansprüche ein systematisches und rechtlich abgesichertes Verfahren vorzusehen, in das die Sozialversicherung eingebunden sein kann ${ }^{70}$ - ohne dass gleichzeitig die Finanzierung von den Bedingungen der betrieblichen Lage abhängig gemacht werden muss.

Umgekehrt sind Anpassungsansprüche nicht notwendig von einer bestimmten Finanzierung abhängig: Warum sollte ein Arbeitgeber nicht verpflichtet werden können, Anpassungen im Rahmen des betrieblich Möglichen zu gewährleisten, auch wo er nicht gleichzeitig für die finanziellen Risiken solcher Entscheidungen aufkommen muss? Anders als Ansprüche auf Finanzierung aus Mitteln Dritter braucht die Anpassung im Betrieb keineswegs von der betrieblichen oder gesellschaftlichen Nützlichkeit abhängig gemacht zu werden. So sollte die Freiheit, ein Sabbatjahr für den Bau eines Hauses oder eine Weltreise zu nehmen, als allein privatnützige Entscheidung zwar privat durch die Beschäftigten finanziert werden müssen; dies

69 Schmid 2012; Hipp 2009, S. 366 f.; Hammer, Rosemann 2012; Käpplinger et al. 2013; siehe auch SPD-Regierungsprogramm 2013-2017, S. 23. Hinsichtlich der »Verwirklichung beruflicher Ziele « ist die Forderung des SPD-Regierungsprogramms nach »Unterstützung « durch die Arbeitsversicherung wohl nicht als Finanzierungsvorschlag in Bezug auf Entgelt und sonstige Kosten zu verstehen; möglicherweise ist dies als Gegenstand zu unterstützender tariflicher Weiterbildungsfonds gemeint (ebd., S. 49).

70 Was insbesondere in Hinblick auf die erforderliche Beratung sinnvoll sein kann; vgl. Schmid 2012, S. 263. 
schließt aber nicht aus, dass der- oder diejenige im betrieblichen Konfliktfall durch einen rechtlichen Anspruch unterstützt wird.

Betriebliche Ansprüche auf Zeit (Auszeit, Arbeitszeitreduzierung oder Arbeitszeitanpassung) müssen auch nicht auf eine fest vorgegebene konkrete Zeitspanne begrenzt werden, wie es aktuell bei der Eltern- und Pflegezeit der Fall ist. ${ }^{71}$ Schließlich sind die möglichen Konflikte hier von anderer Art als bei Fragen der Finanzierung: Hier besteht das Problem nicht in der Ressourcenknappheit, sondern in der Konkurrenz und dem Konflikt von Zeitnutzungswünschen. Zur Lösung solcher Konflikte innerhalb der Belegschaft sowie in der betrieblichen Organisation bedarf es der Festschreibung zwingender Verfahren unter Beteiligung wirkmächtiger Vertreter der jeweiligen Interessen. ${ }^{72}$ Die Benennung konkreter Zeitspannen kann eine Hilfestellung für die Verwirklichung eines Mindestrechts sein, ist darüber hinaus aber nur erforderlich, wo es um die Finanzierung durch Mittel Dritter oder die soziale Sicherung geht.

\section{Weitergehend: Trennung von Finanzierungsanspruch und Bereitstellung der Ressourcen}

Für die Ausgestaltung von Finanzierungsansprüchen kann eine weitere Unterscheidung zwischen Finanzierungsansprüchen und der Bereitstellung von Ressourcen nützlich sein. Denn Rechtsansprüche haben gegenüber Ziehungsrechten und Fonds zwar den Vorteil, dass sie Verteilungsentscheidungen transparent machen können. ${ }^{73}$ Es besteht aber die Gefahr, dass die rechtsgebundenen Entscheidungsverfahren dysfunktional wirken, wenn die Entscheidungsträger/innen Zielkonflikten und Ressourcenbeschränkungen unterliegen, die nichts mit den rechtlichen Vorgaben zu tun haben und deshalb die Selbstbestimmung stärker einschränken als rechtlich zulässig oder erforderlich. So dürfte zum Beispiel für die Mängel bei der Durchsetzung der geltenden arbeitsförderungsrechtlichen Ansprüche auf Weiterbildungsfinanzierung auch die Tatsache verantwortlich sein, dass aktuell die Weiterbildungsförderung durch die Agenturen für Arbeit einer intransparenten und zumindest teilweise falschen Anreizen folgenden »Vermittlungslogik « folgt. ${ }^{74}$

Hier bieten die Modelle für "accounts, credits, savings, vouchers", wie sie im Zusammenhang mit Konten- und Fondsmodellen entwickelt wurden, interessante Anregungen. So könnten die notwendigen Ressourcen in der internen Organisation des jeweiligen Trägers in Form von individuellen »Konten « berechnet und zur Verfügung gestellt werden. Dies wäre Grundlage eines alternativen Modells für die Steuerung und das Management der Weiterbildungsförderung - ohne dass solche

71 \15 BEEG; $\mathbb{S} 2$ 2-4 PflZG.

72 Genauer dazu oben bei Fußnoten $51 \mathrm{ff}$.

73 Siehe dazu oben bei Fußnoten $27 \mathrm{ff}$.

74 Bosch 2010, S. 9, 40.

Leviathan, 41. Jg., 3/2013 
Konten notwendig den Rechtsanspruch der Betroffenen begründen oder begrenzen müssten.

Eine ähnliche Funktion können tarifliche oder andere überbetriebliche »Fonds « erfüllen, wenn es um die Finanzierung von Ansprüchen gegen Arbeitgeber geht. Die Vorteile solcher Fonds liegen nicht nur in der möglichen Dezentralisierung der Steuerung, sondern auch in der kollektiven und überbetrieblichen Refinanzierung von Lasten der Unternehmen. In der deutschen Tarifpolitik gibt es seit den 1990er Jahren bereits Weiterbildungsfonds der Tarifparteien; ${ }^{75}$ der Betriebliche Demografiefonds nach dem »Tarifvertrag Lebensarbeitszeit und Demografie« der Chemieindustrie aus dem Jahre 2008 kann beispielsweise für unterschiedliche Auszeiten genutzt werden. Einen gesetzlichen Rahmen für eine überbetriebliche Verteilung der Arbeitgeberlasten für Auszeiten von Beschäftigten bietet bereits heute das Aufwendungsausgleichsgesetz (AAG) - allerdings eng beschränkt auf die Entgeltfortzahlung im Krankheitsfall und den Mutterschutz. Solche Instrumente erleichtern die Finanzierung von Anpassungen durch die Arbeitgeber, ohne dass sie gleichzeitig die Ansprüche der Betroffenen begründen oder begrenzen.

\section{Anpassung und Finanzierung am Beispiel von Zeitwertkonten}

Trennt man so einerseits die Ansprüche auf Anpassung und auf Finanzierung sowie andererseits die Finanzierungsansprüche von der Ressourcenbereitstellung, wird auch die mögliche Funktion betrieblicher Langzeit(wert)konten im Kontext eines Rechts auf eine selbstbestimmte Erwerbsbiografie deutlich.

Diese Konten können zunächst die Anpassung von Arbeitszeiten an individuelle Bedürfnisse erleichtern. Dies kann durch vertragliche Rechtsansprüche geschehen, die in den Kontenvereinbarungen festzuschreiben wären. Darüber hinaus schafft die Institutionalisierung von Zeitwertkonten faktisch Gelegenheitsstrukturen auch für die Verwirklichung gesetzlicher Zeitanpassungsansprüche im Betrieb: Wo es solche Konten gibt, ist Flexibilität in der betrieblichen Arbeitszeitorganisation mitgedacht, sodass die Geltendmachung entsprechender Rechtsansprüche nicht so schnell auf unüberwindbare arbeitsorganisatorische Barrieren stößt. ${ }^{76}$

Die Hauptfunktion der Konten liegt aus rechtlicher Sicht allerdings darin, dass sie in einer Übergangssituation der finanziellen Absicherung des Lebensunterhalts und der Absicherung gegen sekundäre soziale Risiken dienen können. Die Konten ermöglichen den Beschäftigten das Bruttosparen und damit eine eigene (private) Finanzierung von Anpassungszeiten. Sie verteilen die Finanzierungskosten jedoch zu Lasten der Beschäftigten und sollten deshalb von Gesetzes wegen nur für solche Fälle als Finanzierungsinstrumente vorgesehen werden, in denen die Anpassung allein der Verwirklichung der persönlich-privaten Bedürfnisse der Betroffenen dient. Als Instrumente zur Finanzierung von Anpassungen, die im betrieblichen oder gesellschaftlichen Interesse liegen (zum Beispiel gesellschaftlich notwendige/legitime

75 Bosch 2010, S. $19 \mathrm{ff}$.

76 Vgl. Wotschack et al. 2011. 
Sorgearbeit, gesundheitsbedingte Leistungseinschränkungen), sind sie ungeeignet. ${ }^{77}$ Für solche Situationen erscheint eine unmittelbare Leistungsgewährung aus Steuer- oder Sozialversicherungsmitteln sinnvoll und legitim. Dadurch lässt sich auch ein gerechter sozialer Ausgleich gewährleisten. Dementsprechend sollte die Finanzierung der Freistellung für Angehörigenpflege nicht der privaten Finanzierung durch Zeitwertkonten überantwortet werden, wie es derzeit noch durch das Familienpflegezeitgesetz (FamPflZG) von 2012 geschieht.

\section{Zusammenfassung und Ergebnisse}

Das Konzept der Ziehungsrechte hat wichtige Impulse für die Entwicklung erweiterter Normalitätsannahmen für das Arbeits- und Sozialrecht gesetzt; es soll insbesondere eine am tatsächlichen Lebensverlauf orientierte Gestaltung des Rechts ermöglichen. Das »Recht auf eine selbstbestimmte Erwerbsbiografie « kann als ein Versuch gelesen werden, dieses Konzept vor dem Hintergrund einer allgemeinen bürgerrechtlichen Betrachtung der Erwerbsarbeit der deutschen Rechtsordnung gemäß auszuformulieren. Ein Verdienst solcher übergreifender Konzepte besteht darin, dass sie über die zahlreich bestehenden Einzelregelungen und Beschäftigungsformen hinweg die Chance einer zusammenfassenden Betrachtung unabhängig von Erwerbs- und Beschäftigungsformen eröffnen. ${ }^{78}$ Sie erinnern daran, dass unterschiedliche Rechtsbereiche und Ansprüche der Verwirklichung desselben rechtlich geschützten Interesses dienen können. Entsprechend verbindet das Recht auf eine selbstbestimmte Erwerbsbiografie Anforderungen an Unternehmen, Sozialversicherung und den Staat konzeptionell miteinander.

Der vorliegende Beitrag sollte aufzeigen, welche Folgen sich ergeben, wenn man das subjektive Recht als Bündel unterschiedlicher Ansprüche begreift, bei deren Regelung jeweils unterschiedliche Verteilungsentscheidungen zu treffen und unterschiedliche Verfahren in Betracht zu ziehen sind. So kann auch der »Gegensatz zwischen der Genauigkeit und empirisch sorgfältig fundierten Subtilität des SupiotBerichts und der relativen Unbestimmtheit « der Durchsetzung ${ }^{79}$ aufgelöst werden.

$\mathrm{Zu}$ unterscheiden sind dabei insbesondere Anpassungsansprüche, die sich gegen den Arbeitgeber richten und die im geltenden Recht bereits grundsätzlich anerkannt sind, von Finanzierungsansprüchen, die sich gegen unterschiedliche Träger und Vermögensmassen richten können und die im geltenden Recht noch nicht nach einer erkennbaren Systematik auf das Recht auf eine selbstbestimmte Erwerbsbiografie ausgerichtet sind.

Die Vorteile einer solchen Unterscheidung bestehen einerseits darin, dass differenzierte Anspruchsvoraussetzungen und damit Verteilungsentscheidungen unab-

77 Zumal die Eignung von Zeitwertkonten für eine selbstbestimmte Erwerbsbiografie auch dadurch begrenzt wird, dass auf solchen Konten eine Vielzahl von Zeitverwendungswünschen lasten; Seifert et al. 2013.

78 Mückenberger 2007, S. 196.

79 So Castel 2011, S. 102 in Bezug auf die Frage der Finanzierung. 
hängig voneinander diskutiert werden können. Zugleich können die Ansprüche aus unterschiedlichen Verfahren miteinander verbunden werden. Der Beitrag vertritt insofern die These, dass das Recht auf eine selbstbestimmte Erwerbsbiografie einer prozeduralen Ausgestaltung der Ansprüche bedarf. Nur die Verbindung entsprechender Ansprüche mit reflexiv wirkenden Verfahren vermag das Recht tatsächlich wirksam zu machen. Bei dieser Betrachtung von Recht als »Verfahren « offenbart sich eine weitere innere Logik der Unterscheidung zwischen arbeitsrechtlichen Ansprüchen für betriebliche Konflikte und den (meist sozialversicherungsrechtlichen) Finanzierungsansprüchen: Beide Gebiete beziehen sich auf unterschiedliche Akteurinnen und Akteure, unterschiedliche Ressourcen und somit unterschiedliche Konfliktpotenziale. Um durchgesetzt werden zu können, bedarf jedes Recht und jeder Anspruch eigener wirksamer Verfahren der Implementation und Mobilisierung. ${ }^{80}$

Im Einzelnen wird für Verfahren zur Durchsetzung von Anpassungsansprüchen eine prozedurale Regelung von »betrieblichen Übergangsmanagements « vorgeschlagen, die betriebliche Interessenvertretungen genau wie externe Akteure (insbesondere aus den Sozialversicherungen) einbezieht. Für die Geltendmachung von Finanzierungsansprüchen wiederum wird eine Trennung des Anspruchs von den Ressourcenzuweisungen vorgeschlagen, die verhindern kann, dass die Durchsetzung von Rechtsansprüchen an sach- (und meist auch rechts-)widrigen Zielkonflikten und Ressourcenbeschränkungen scheitert.

In vielen sozialwissenschaftlichen Debatten wird das Recht nur als äußeres Orientierungssystem und feststehendes Regelwerk betrachtet. Für die Rechtswissenschaften ist das Recht jedoch auch ein umkämpftes Entscheidungssystem für Konflikte. Der vorliegende Text soll einen sozialwissenschaftlichen Blick auf diese Funktionsbedingungen des Rechts anregen.

\section{Literatur}

Arthurs, Harry 2011. »Labour law after labour «, in The idea of labour law, hrsg. v. Davidov, Guy; Langille, Brian, S. 13-29. Oxford: Oxford University Press.

Blanke, Thomas; Bleses, Peter 2005. »Flexicurity im Arbeits- und Sozialleistungsrecht «, in Flexicurity. Die Suche nach Sicherheit in der Flexibilität, hrsg. v. Kronauer, Martin; Linne, Gudrun, S. 365-384. Berlin: edition sigma.

Blankenburg, Erhard 1980. »Mobilisierung von Recht ", in Zeitschrift für Rechtssoziologie 1, 1, S. 33-64.

Bonvin, Jean-Michel 2012. »Individual working lives and collective action. An introduction to capability for work and capability for voice", in Transfer - European Review of Labour and Research 18, 1, S. 9-18.

Bosch, Gerhard 2010. In Qualifizierung investieren - ein Weiterbildungsfonds für Deutschland. Expertise im Auftrag der Friedrich-Ebert-Stiftung. Bonn: Friedrich-Ebert-Stiftung.

Bryde, Brun-Otto 1984. "Artikel 12 Grundgesetz - Freiheit des Berufs und Grundrecht der Arbeit", in Neue Juristische Wochenschrift, S. 2177-2184.

Castel, Robert 2011. Die Krise der Arbeit. Neue Unsicherheiten und die Zukunft des Individuums. Hamburg: Hamburger Edition.

Deakin, Simon; Rogowski, Ralf 2011. "Reflexive labour law, capabilities and the future of social Europe", in Transforming European employment policy. Labour market transitions and the

80 Zu diesem Begriff Blankenburg 1980; für das Arbeitsrecht Kocher 2009. 
promotion of capability, hrsg. v. Rogowski, Ralf et al., S. 229-254. Cheltenham, Northampton: Edward Elgar.

Drouin, Renée-Claude 2009. ")Capacitas and capabilities in international labour law", in Capacitas. Contract law and the institutional preconditons of a market economy, hrsg. v. Deakin, Simon; Supiot, Alain, S. 141-160. Oxford: Hart Publishing.

Durkalski, John A. 2009. "Fixing economic flexibilization. A role for flexible work laws in the workplace policy agenda ", in Berkeley Journal of Employment and Labor Law 30, 2, S. 381-403.

Eichenhofer, Eberhard 2012. »Soziales Recht - Bemerkungen zur Begriffsgeschichte ", in Soziales Recht 2, S. 76-83.

Europäische Kommission 2006. Grünbuch: Ein modernes Arbeitsrecht für die Herausforderungen des 21. Jahrhunderts. KOM(2006) 708 endgültig. Brüssel.

Europäische Kommission 2007. Gemeinsame Grundsätze für den Flexicurity-Ansatz herausarbeiten: Mehr und bessere Arbeitsplätze durch Flexibilität und Sicherheit. KOM(2007) 359 endgültig. Brüssel.

Europäische Kommission 2012. Einen arbeitsplatzintensiven Aufschwung gestalten. KOM(2012) 173 endgültig. Brüssel.

Expertenkommission Finanzierung Lebenslangen Lernens 2004. Finanzierung Lebenslanges Lernen: Der Weg in die Zukunft. Berlin.

Freedland, Mark R.; Kountouris, Nicola 2011. »The legal characterization of personal work relations and the idea of labour law", in The idea of labour law, hrsg. v. Davidov, Guy; Langille, Brian, S. 190-208. Oxford: Oxford University Press.

Fudge, Judy 2007. "The new discourse of labor rights: from social to fundamental rights? «, in Comparative Labor Law \& Policy Journal 29, S. 29-66.

Giesecke, Johannes; Heisig, Jan P. 2010. Höheres Risiko für Geringqualifizierte. Wie sich die berufliche Mobilität in Deutschland verändert hat. WZBrief Arbeit 07/Oktober 2010. Berlin: Wissenschaftszentrum Berlin für Sozialforschung.

Hammer, Angelika; Rosemann, Martin 2012. Finanzierungspotenziale bei der Weiterentwicklung der gesetzlichen Arbeitslosenversicherung zur Arbeitsversicherung. Expertise für die Friedrich-Ebert-Stiftung. Bonn: Friedrich-Ebert-Stiftung.

Hipp, Lena 2009. »Weiter mit Weiterbildung! Von der Arbeitslosen- zur Beschäftigungsversicherung ", in WSI-Mitteilungen 62, 7, S. 362-368.

Käpplinger, Bernd; Kulmus, Claudia; Haberzeth, Erik 2013. Weiterbildungsbeteiligung. Anforderungen für eine Beschäftigungs- und Arbeitsversicherung. Expertise für die FriedrichEbert-Stiftung. Bonn: Friedrich-Ebert-Stiftung.

Käufer, Katja 2002. Weiterbildung im Arbeitsverhältnis. Baden-Baden: Nomos.

Klammer, Ute 2005. "Flexicurity aus der Perspektive des Lebenslaufs «, in Flexicurity. Die Suche nach Sicherheit in der Flexibilität, hrsg. v. Kronauer, Martin; Linne, Gudrun, S. 249-273. Berlin: edition sigma.

Klammer, Ute 2010. »Flexibilität und Sicherheit im individuellen (Erwerbs-) Lebensverlauf. Zentrale Ergebnisse und politische Empfehlungen aus der Lebenslaufforschung der European Foundation ", in Soziale Lebenslaufpolitik, hrsg. v. Naegele, Gerhard; Bertermann, Britta, S. 675-710. Wiesbaden: VS Verlag für Sozialwissenschaften.

Kocher, Eva 2007. "Gestaltungs- und Verhandlungsrechte von Beschäftigten ", in WSI-Mitteilungen 60, 8, S. 434-440.

Kocher, Eva 2009. Effektive Mobilisierung von Beschäftigtenrechten. Das Arbeitsrecht in der betrieblichen Praxis. Düsseldorf: Hans-Böckler-Stiftung.

Kocher, Eva 2012. "Solidarität und Menschenrechte - Zwei verschiedene Welten? «, in Erzählungen vom Konstitutionalismus. Festschrift für Günter Frankenberg, hrsg. v. Lindemann, Helena et al., S. 151-162. Baden-Baden: Nomos.

Kocher, Eva; Groskreutz, Henning; Nassibi, Ghazaleh; Paschke, Christian; Schulz, Susanne; Welti, Felix; Wenckebach, Johanna; Zimmer, Barbara 2013. Das Recht auf eine selbstbestimmte Erwerbsbiografie. Arbeits- und sozialrechtliche Regulierung für Übergänge im Lebenslauf. Ein Beitrag zu einem Sozialen Recht der Arbeit. Baden-Baden: Nomos.

Kocher, Eva; Welti, Felix 2010. »Autonomie und soziale Sicherheit - Anforderungen an arbeitsund sozialrechtliche Regulierung ", in WSI-Mitteilungen 63, 6, S. 299-305. 
Kocher, Eva; Welti, Felix 2013. Wie lässt sich ein Anspruch auf Weiterbildung rechtlich gestalten? Rechtliche Instrumente im Arbeits- und Sozialrecht. Expertise für die Friedrich-Ebert-Stiftung. Bonn: Friedrich-Ebert-Stiftung.

Kohte, Wolfhard 2010 a. »Das betriebliche Eingliederungsmanagement - Ein doppelter Suchprozess ", in WSI-Mitteilungen 63, 7, S. 374-377.

Kohte, Wolfhard 2010 b. »Return to work - Europäische Impulse und deutsche Handlungsmöglichkeiten ", in Geschlechtergerechtigkeit: Festschrift für Heide Pfarr, hrsg. v. HohmannDennhardt, Christine; Körner, Marita; Zimmer, Reingard, S. 489-503. Baden-Baden: Nomos.

Kruppe, Thomas 2013. Organisation und Finanzierung von Qualifizierung und Weiterbildung im Lebensverlauf. Expertise für die Friedrich-Ebert-Stiftung. Bonn: Friedrich-Ebert-Stiftung.

Leisering, Lutz 2003. "Government and the life course", in Handbook of the life course, hrsg. v. Mortimer, Jeylan T.; Shanahan, Michael J., S. 205-225. New York: Springer.

Mückenberger, Ulrich 2007. »Ziehungsrechte. Ein zeitpolitischer Weg zur >Freiheit in der Arbeit«", in WSI-Mitteilungen 60, 4, S. 195-201.

Naegele, Gerhard; Bertermann, Britta. Hrsg. 2010. Soziale Lebenslaufpolitik. Wiesbaden: VS Verlag für Sozialwissenschaften.

Nassibi, Ghazaleh 2012. "Die Durchsetzung der Ansprüche auf Schaffung behinderungsgerechter Arbeitsbedingungen ", in Neue Zeitschrift für Arbeitsrecht 13, S. 720-725.

Natzel, Ivo 2010. »Unwissenheit ist freiwilliges Unglück. Qualifizierung als Herausforderung beider Arbeitsvertragsparteien ", in Betriebs-Berater 12, S. 697-702.

Niehaus, Mathilde; Vater, Gudrun E. 2010. "Aktueller Stand der Umsetzung des Betrieblichen Eingliederungsmanagements", in Feblzeiten-Report 2010. Vielfalt managen: Gesundheit fördern - Potenziale nutzen. Zahlen, Daten, Analysen aus allen Branchen der Wirtschaft, hrsg. v. Badura, Bernhard; Schröder, Helmut; Klose, Joachim, S. 189-205. Berlin: Springer.

Nussbaum, Martha 2008. "Menschenrechte und Fähigkeiten über Grenzen hinweg ", in Transnationale Menschenrechte. Schritte zu einer weltweiten Verwirklichung der Menschenrechte, hrsg. v. Richter, Ingo, S. 61-83. Opladen, Farmington Hills: Verlag Barbara Budrich.

Paschke, Christian 2012. "Zeitsouveränität durch Anpassung der Arbeitszeitlage an die persönlichen Bedürfnisse. Individuelle Zeitansprüche und kollektive Mitbestimmung ", in Arbeit und Recht 1, S. 11-16.

Perulli, A. 2003. Travail économiquement dépendant / parasubordination: les aspects juridiques, sociales et économiques. http://www.metiseurope.eu/content/pdf/n8/7_parasubordination.pdf (Zugriff vom 15.06.2013).

Pfarr, Heide 2000. »Soziale Sicherheit und Flexibilität: Brauchen wir ein >Neues Normalarbeitsverhältnis<? «, in WSI-Mitteilungen 53, 5, S. 279-283.

Radbruch, Gustav 1957. Der Mensch im Recht. Göttingen: Vandenhoeck \& Ruprecht.

Sachverständigenkommission 2006. Siebter Familienbericht: Familie zwischen Flexibilität und Verlässlichkeit - Perspektiven für eine lebenslaufbezogene Familienpolitik. Bundestagsdrucksache 16/1360.

Schmid, Günther 2011. Übergänge am Arbeitsmarkt. Arbeit, nicht nur Arbeitslosigkeit versichern. Berlin: edition sigma.

Schmid, Günther 2012. »Von der Arbeitslosen- zur Arbeitsversicherung «, in Leviathan 40, 2 , S. 248-270.

Schneider, Hans-Peter 1985. »Artikel 12 GG - Freiheit des Berufs und Grundrecht der Arbeit «, in Veröffentlichungen der Vereinigung deutscher Staatsrechtslehrer 43, S. 7-47.

Seifert, Achim 2011. ") Von der Person zum Menschen im Recht $`$ Zum Begriff des sozialen Rechts bei Hugo Sinzheimer ", in Soziales Recht 3, S. 62-73.

Seifert, Hartmut; Kümmerling, Angelika; Riedmann, Arnold 2013. »Langzeitkonten - überschätzte Erwartungen einer biografieorientierten Zeitpolitik? «, in WSI-Mitteilungen 66, 2, S. 133-142.

Sen, Amartya K. 2010. Die Idee der Gerechtigkeit. München: C. H. Beck.

Supiot, Alain 2001. Beyond employment. Changes in work and the future of labour law in Europe. Oxford: Oxford University Press.

Supiot, Alain 2009. »En guise de conclusion: la capacité, une notion à haut potentiel «, in Capacitas. Contract law and the institutional preconditons of a market economy, hrsg. v. Deakin, Simon; Supiot, Alain, S. 161-171. Oxford: Hart Publishing. 
Teubner, Gunther; Willke, Helmut 1984. »Kontext und Autonomie: Gesellschaftliche Selbststeuerung durch reflexives Recht ", in Zeitschrift für Rechtssoziologie 5, S. 4-35.

Welti, Felix; Kocher, Eva; Paschke, Christian; Nassibi, Ghazaleh; Wenckebach, Johanna; Zimmer, Barbara 2012. "Den sozialstaatlichen Pfad wieder finden: Arbeit, nicht nur Arbeitslosigkeit versichern. Anmerkungen zu einem Vorschlag von Günther Schmid", in Soziale Sicherheit 5, S. 184-188.

Wilthagen, Ton; Tros, Frank 2004. »The concept of sflexicurity : a new approach to regulating employment and labour markets", in Transfer. European Review of Labour and Research 10, 2, S. 166-186.

Wotschack, Philip 2011. »Working-time options over the life course: challenges and company practices ", in Transforming European employment policy. Labour market transitions and the promotion of capability, hrsg. v. Rogowski, Ralf et al., S. 96-114. Cheltenham, Northampton: Edward Elgar.

Wotschack, Philip; Scheier, Franziska; Schulte-Braucks, Philipp; Solga, Heike 2011. »Zeit für Lebenslanges Lernen. Neue Ansätze der betrieblichen Arbeitszeit- und Qualifizierungspolitik«, in WSI-Mitteilungen 64, 10, S. 541-547. 
Zusammenfassung: In diesem Beitrag wird davon ausgegangen, dass eine Lebenslaufperspektive für das Arbeits- und Sozialrecht neue Normalitätsannahmen verlangt und schlägt als Konzept hierfür das »Recht auf eine selbstbestimmte Erwerbsbiografie « vor. Zur Verwirklichung dieses Rechts seien Ansprüche auf Anpassung des Arbeitsverhältnisses grundsätzlich zu trennen von Finanzierungsansprüchen, und die jeweiligen Ansprüche seien prozedural auszugestalten, also mit reflexiv wirkenden Verfahren zu verbinden.

Stichworte: Arbeits- und Sozialrecht, Lebenslaufperspektive, Ziehungsrechte, Sorgearbeit, Weiterbildung, Arbeitsversicherung

\section{The right to an autonomous occupational biography: a procedural view on social drawing rights}

Summary: A life course perspective on employment and social security laws presupposes new normative ideas of "normality « in employment. The text proposes the concept of a right to an autonomous occupational biography as a comprehensive concept. In order to achieve this, entitlements arising from the adaptation of the employment relationship need to be fundamentally divorced from financial entitlements. Procedurally, relevant entitlements should be linked to reflexive enforcement procedures.

Keywords: labour law and social security, life course perspective, social drawing rights, private care, life-long learning, employment insurance

Autorin

Prof. Dr. Eva Kocher

Juristische Fakultät

Europa-Universität Viadrina

Große Scharrnstr. 59

15230 Frankfurt (Oder)

kocher@europa-uni.de 